\title{
Myocardial Motion Analysis From B-Mode Echocardiograms
}

\author{
Michael Sühling, Student Member, IEEE, Muthuvel Arigovindan, Christian Jansen, Patrick Hunziker, and \\ Michael Unser, Fellow, IEEE
}

\begin{abstract}
The quantitative assessment of cardiac motion is a fundamental concept to evaluate ventricular malfunction. We present a new optical-flow-based method for estimating heart motion from two-dimensional echocardiographic sequences. To account for typical heart motions, such as contraction/expansion and shear, we analyze the images locally by using a local-affine model for the velocity in space and a linear model in time. The regional motion parameters are estimated in the least-squares sense inside a sliding spatiotemporal B-spline window. Robustness and spatial adaptability is achieved by estimating the model parameters at multiple scales within a coarse-to-fine multiresolution framework. We use a wavelet-like algorithm for computing B-spline-weighted inner products and moments at dyadic scales to increase computational efficiency. In order to characterize myocardial contractility and to simplify the detection of myocardial dysfunction, the radial component of the velocity with respect to a reference point is color coded and visualized inside a time-varying region of interest. The algorithm was first validated on synthetic data sets that simulate a beating heart with a speckle-like appearance of echocardiograms. The ability to estimate motion from real ultrasound sequences was demonstrated by a rotating phantom experiment. The method was also applied to a set of in vivo echocardiograms from an animal study. Motion estimation results were in good agreement with the expert echocardiographic reading.
\end{abstract}

Index Terms-Echocardiography, motion estimation, timevarying deformable model.

\section{INTRODUCTION}

$\mathbf{E}$ CHOCARDIOGRAPHY is an effective imaging modality that enables clinicians to study the shape, size, and dynamics of the heart. It is noninvasive, relatively inexpensive, and fairly simple to use. The analysis of ventricular motion, in particular, provides an efficient means to evaluate the degree of ischemia and infarction [1]-[3]. In clinical practice, the analysis mainly relies on visual inspection or manual measurements by experienced cardiologists. Manual methods are tedious and time consuming, and visual assessment leads to qualitative and subjective diagnoses that suffer from a considerable inter- and intra-observer variability. Therefore, an automated,

Manuscript received June 15, 2003; revised December 17, 2003. This work was supported in part by the Swiss National Science Foundation under Grant 3200-059517.99 and in part by the Swiss Heart Foundation. The associate editor coordinating the review of this manuscript and approving it for publication was Dr. Tamas Sziranyi.

M. Sühling, M. Arigovindan, and M. Unser are with the Biomedical Imaging Group, Swiss Federal Institute of Technology Lausanne (EPFL), CH-1015 Lausanne, Switzerland (e-mail: michael.suehling@epfl.ch; muthuvel.arigovindan@epfl.ch; michael.unser@epfl.ch).

C. Jansen and P. Hunziker are with the University Hospital Basel, CH-4031 Basel, Switzerland (e-mail: christian.jansen@unibas.ch; phunziker@uhbs.ch). Digital Object Identifier 10.1109/TIP.2004.838709 computer-based analysis is highly desirable to obtain more objective and quantitative diagnoses.

Several approaches have been proposed to quantify heart motion from two-dimensional (2-D) echocardiograms. One approach consists of segmenting and tracking myocardial borders using active contours [4]-[6] or active appearance models [7]. However, motion information is only obtained for myocardial borders which are often poorly defined, especially in the case of the epicardial border. A different approach is to estimate motion for the entire image content; optical flow methods, in particular, have led to promising results: Mailloux et al. [8] extended the optical flow algorithm of Horn-Schunk [9] by adding a linearity constraint to the motion field. Zini et al. [10] added an additional incompressibility constraint. A popular optical flow algorithm is the Lucas-Kanade method [11], which estimates the motion locally, assuming that the velocity field is constant within a window. This method was applied to ultrasound by Chunke et al. [12]. An evaluation of different optical flow methods applied to echocardiograms can be found in [13].

In this paper, we propose a novel optical-flow-based algorithm that is tuned for the analysis of ventricular wall motion from dynamic B-mode echocardiograms. Inspired by the Lucas-Kanade method [11], we use a local motion model inside a sliding spatiotemporal window. We use a local-affine model for the velocity in space that allows to describe typical heart motions such as rotation, contraction/expansion and shear. The motion parameters are estimated in the weighted least-squares sense inside the sliding spatiotemporal window. Instead of working at a fixed scale, we consider estimation windows of increasing sizes and develop a multiresolution strategy to improve the estimation of large motions and to reduce the sensitivity to noise. We introduce a B-spline weighting scheme that has important computational advantages for multiscale processing. In particular, we develop a wavelet-like multiresolution implementation. We also add a temporal linear component to our motion model. This leads to a more robust motion estimation which combines the information from multiple frames. It also yields an additional acceleration parameter-a useful indicator of heart dynamics.

Since the estimated velocity data itself is not of direct use for the clinician, it has to be processed and visualized properly to facilitate the diagnosis. In order to focus on the relevant regions of the heart, the motion information is only displayed inside a user-defined region of interest (ROI) that typically corresponds to the myocardium. In order to follow the movement of the myocardium, the ROI contour is automatically tracked 
in time by using the estimated velocity field. Robustness of the tracking process is achieved by using a spline representation of the ROI contours that is fitted in the least-squares sense to the estimated motion field. To extract myocardial contractility information from the overall motion field, we compute the radial component of the estimated velocity field with respect to the ROI centroid (center of gravity). This radial velocity component is then color coded and superimposed on the original image sequence in a semitransparent fashion that is similar to tissue Doppler imaging. The color display allows a more intuitive and simplified identification of regions with abnormal motion patterns. As additional information, the motion trajectory of the ROI centroid, the principal axes of inertia and the area size of the ROI can also be displayed.

The paper is organized as follows. We describe the motion estimation algorithm in Section II. The ROI tracking and radial motion visualization is presented in Section III. The algorithm is validated in Section IV. First, we apply it to synthetic data that simulates the characteristics of a beating heart and compare it with alternative motion estimation approaches. In particular, we test its robustness by simulating the ultrasonic image formation to generate characteristic speckle noise. Second, we demonstrate the ability of the proposed method to analyze motion from real echocardiograms by performing an experiment with a tissue mimicking phantom that is described in Section IV-B. Finally, in Section IV-C, we validate the algorithm on a set of clinical echocardiograms obtained from an animal study. Data sets that were acquired before and after an artificially induced infarction were analyzed and compared.

\section{LoCAL-Affine, Multiscale Motion Estimation}

Several methods have been proposed to quantify heart motion from 2-D echocardiograms. Special attention has been paid to optical flow methods which have led to promising results [13]. Gradient-based optical flow estimation relies on the assumption that the intensity of a particular point in a moving pattern does not change with time. Let $I(x, y, t)$ denote the intensity of pixels at location $\mathbf{r}=(x, y)$ and time $t$ in an image sequence. The constant intensity assumption can be expressed as [9]

$$
I_{x}(\mathbf{r}, t) u(\mathbf{r}, t)+I_{y}(\mathbf{r}, t) v(\mathbf{r}, t)+I_{t}(\mathbf{r}, t)=0
$$

where $I_{x}, I_{y}$, and $I_{t}$ denote the spatial and temporal derivatives of the image intensity. To obtain accurate numerical derivative estimates, we use cubic spline interpolation [14] in space and time. The velocities $u$ and $v$ are, respectively, the $x$ and $y$ components of the optical flow that we wish to estimate. Since (1) is a single equation in two unknowns $u$ and $v$, it cannot be solved uniquely without introducing additional constraints.

\section{A. Local-Affine Velocity in Space Time}

A popular optical flow algorithm is the Lucas-Kanade method [11], which was applied to ultrasound in at least two studies [13], [12]. The method estimates the motion locally assuming it to be constant within a spatial window. Since typical heart motions are given by rotation, expansion, contraction, and shear, we use a local-affine model for the motion in space. Additionally, we introduce a linear model for the velocity along the time direction. This allows us to capture local accelerations in time better than by a locally constant model. Another advantage is that we can base our estimation on multiple frames around a given time point which is more robust than using only two frames as many classical optical flow methods do. Let $\left(x_{0}, y_{0}, t_{0}\right)$ denote the center of a small spatiotemporal image region $\Omega$. Then the spatiotemporal-affine model is defined as

$$
\left(\begin{array}{l}
u(x, y, t) \\
v(x, y, t)
\end{array}\right)=\left(\begin{array}{l}
u_{0} \\
v_{0}
\end{array}\right)+\left(\begin{array}{lll}
u_{x} & u_{y} & u_{t} \\
v_{x} & v_{y} & v_{t}
\end{array}\right)\left(\begin{array}{l}
x-x_{0} \\
y-y_{0} \\
t-t_{0}
\end{array}\right) .
$$

The parameters $u_{0}$ and $v_{0}$ correspond to the velocity at the center point $\left(x_{0}, y_{0}, t_{0}\right) ; u_{x}, u_{y}, u_{t}, v_{x}, v_{y}$, and $v_{t}$ are the first order spatial and temporal derivatives of $u$ and $v$, respectively. The derivatives are assumed to be constant within the local neighborhood.

The submatrix of spatial derivatives

$$
\mathbf{J}=\left(\begin{array}{ll}
u_{x} & u_{y} \\
v_{x} & v_{y}
\end{array}\right)
$$

describes the local variation of the velocity field in space. It can be decomposed into two terms such that $\mathbf{J}=\mathbf{R}+\mathbf{D}$, where

$$
\begin{aligned}
\mathbf{R} & =\left(\begin{array}{cc}
0 & -\frac{v_{x}-u_{y}}{2} \\
\frac{v_{x}-u_{y}}{2} & 0
\end{array}\right) \\
\text { and } \mathbf{D} & =\left(\begin{array}{cc}
u_{x} & \frac{v_{x}+u_{y}}{2} \\
\frac{v_{x}+u_{y}}{2} & v_{y}
\end{array}\right) .
\end{aligned}
$$

The antisymmetric matrix $\mathbf{R}$ corresponds to a rigid, rotational velocity field that leaves the local tissue area unchanged. The angular velocity is given by $\omega=\left(v_{x}-u_{y}\right) / 2$, which is equal to one half of the curl of the velocity field. The second, symmetric term $\mathbf{D}$ accounts for the deformation of the heart tissue, both contraction/expansion and shear. The components on the principal diagonal describe a dilation along the coordinate axes, whereas the off-diagonal components correspond to a shearing.

We estimate the motion components at $\left(x_{0}, y_{0}, t_{0}\right)$ by minimizing the weighted least-squares criterion

$$
\int_{\mathbb{R}^{3}} w\left(x-x_{0}, y-y_{0}, t-t_{0}\right)\left(I_{x} u+I_{y} v+I_{t}\right)^{2} d x d y d t .
$$

The symmetric window function $w$ gives more weight to constraints at the center of the local spatiotemporal region than to those at the periphery. A well-suited window function is $w(x, y, t)=\beta^{n}(x) \beta^{n}(y) \beta^{n}(t)$, where $\beta^{n}$ is the symmetrical $\mathrm{B}$-spline of degree $n \in \mathbb{N}$ [14]. B-splines rapidly converge to Gaussians when their degree increases, which ensures isotropy of the window in multiple dimensions. Varying the B-spline degree also allows to change the size of the window function. Additionally, the B-splines satisfy a two-scale equation which leads to an efficient computation of B-spline-weighted inner products at dyadic scales by using a wavelet-like algorithm (cf. Section II-B).

By differentiating (3) with respect to each of the eight unknown parameters, we obtain a symmetric linear system $\mathbf{A v}=$ b in terms of local moments of the spatial and temporal derivatives of $I$, as defined in (9). The coefficients of this system of 
equations at a given position $\left(x_{0}, y_{0}, t_{0}\right)$ are of the form shown in (4) at the bottom of the page, where $0 \leq p+q+r \leq 2$ and $f$ represents the functions $I_{x}^{2}, I_{y}^{2}, I_{x} I_{y}, I_{x} I_{t}$, or $I_{y} I_{t}$.

\section{B. Multiresolution Moments Computation}

It is obviously difficult to estimate large motions at fine scales. Small window functions may lead to aliasing artifacts if the motion is large. To reduce this problem, we apply a coarse-to-fine strategy in space. At each spatial scale $j$, we use dilated and shifted versions of the window function

$$
\begin{aligned}
w^{(j)}\left(x-x_{0}, y-y_{0}, t-t_{0}\right) & \\
= & w\left(\frac{x-2^{j} x_{0}}{2^{j}}, \frac{y-2^{j} y_{0}}{2^{j}}, t-t_{0}\right) .
\end{aligned}
$$

The window functions at scale $j$ are dilated by the factor $2^{j}$. They are spaced at a distance of $2^{j}$ pixels which corresponds to a subsampling of the inner products (4) by $2^{j}$ in the spatial dimensions. Computing inner products with large windows is computationally expensive; however, inspired by the framework of wavelets [15], [16], we have developed a multichannel, Mallat-like algorithm to compute the local moments (4) recursively within a multiresolution framework [17]. Since B-splines satisfy a two-scale equation [14], the relevant moments at successive scales can be computed iteratively as

$$
\begin{aligned}
m_{p, q, r}^{(j+1)} & \left(x_{0}, y_{0}, t_{0}\right) \\
& =\sum_{k=0}^{p} \sum_{l=0}^{q}\left(h_{p, k}^{(j)} h_{q, l}^{(j)} * m_{k, l, r}^{(j)}\right)\left(2 x_{0}, 2 y_{0}, t_{0}\right) \\
& =\sum_{k=0}^{p} \sum_{l=0}^{q} \sum_{m, n} h_{p, k}^{(j)}(m) h_{q, l}^{(j)}(n) m_{k, l, r}^{(j)}\left(2 x_{0}-m, 2 y_{0}-n, t_{0}\right) .
\end{aligned}
$$

The one-dimensional two-scale filters

$$
h_{p, k}^{(j)}=2^{j(p-k)} h_{p, k}
$$

are scaled versions of basic filters $h_{p, k}$ and are applied separately in the $x$ - and $y$ directions. The corresponding two-scale filters $h_{p, k}$ for the cubic B-spline $(n=3)$ up to order $p=2$ are given in Table I. Practically, this means that the moments are first computed at the finest scale (initialization) and that the counterparts at successive coarser scales are obtained by multichannel filtering and summation as in (6). For further details, we refer to [17].

\section{Coarse-to-Fine Multiscale Strategy}

Our coarse-to-fine multiresolution strategy is sketched in Fig. 1 and works as follows. First, the local linear system (9) is set up and solved at each spatiotemporal position of the sliding window at the coarsest spatial scale. The motion vectors are
TABLE I

TWO-SCALE FILTERS $h_{p, k}$ UP TO ORDER $p=2$ FOR $\beta^{3}$

\begin{tabular}{c||c|c|c|c|c}
\hline$l$ & -2 & -1 & 0 & 1 & 2 \\
\hline \hline$h_{0,0}(l)$ & $\frac{1}{8}$ & $\frac{4}{8}$ & $\frac{6}{8}$ & $\frac{4}{8}$ & $\frac{1}{8}$ \\
\hline$h_{1,0}(l)$ & $-\frac{1}{4}$ & $-\frac{2}{4}$ & 0 & $\frac{2}{4}$ & $\frac{1}{4}$ \\
\hline$h_{1,1}(l)$ & $\frac{1}{8}$ & $\frac{4}{8}$ & $\frac{6}{8}$ & $\frac{4}{8}$ & $\frac{1}{8}$ \\
\hline$h_{2,0}(l)$ & $\frac{1}{2}$ & $\frac{1}{2}$ & 0 & $\frac{1}{2}$ & $\frac{1}{2}$ \\
\hline$h_{2,1}(l)$ & $-\frac{1}{2}$ & $-\frac{2}{2}$ & 0 & $\frac{2}{2}$ & $\frac{1}{2}$ \\
\hline$h_{2,2}(l)$ & $\frac{1}{8}$ & $\frac{4}{8}$ & $\frac{6}{8}$ & $\frac{4}{8}$ & $\frac{1}{8}$ \\
\hline
\end{tabular}

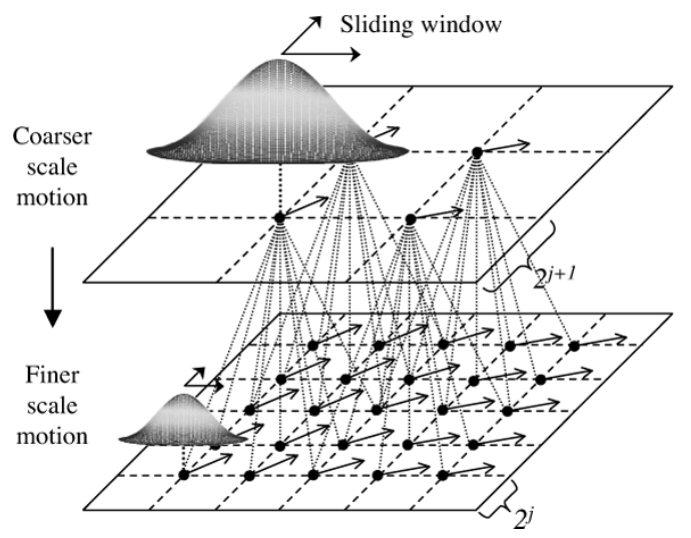

Fig. 1. Coarse-to-fine multiscale strategy in space.

then transferred to the next finer resolution level as initial estimates by using linear interpolation to obtain parameter values on intermediate grid locations. The transferred parameters are then re-estimated if the coarser-scale motion does not already exceed a scale-dependent size. A coarser-scale estimate is replaced only if the following conditions are met.

1) The local linear system has to be well conditioned, i.e., the condition number is below a predefined limit. Its size corresponds to the level above which a linear system is considered to be singular. In the experiments, the value is 2000.

2) The normalized residual error

$$
e=\frac{\|\mathbf{A} \mathbf{v}-\mathbf{b}\|_{l_{2}}}{\left\|w^{(j)}\right\|_{l_{1}}}
$$

is smaller than the corresponding one at the next coarser scale.

3) The length of the estimated central motion vector does not exceed a scale-dependent limit [see (9) at the bottom of the next page].

A motion estimate is set to zero if the local average of the time derivative $\left\langle w^{(j)}, I_{t}^{2}\right\rangle$ is smaller than a predefined noise level. As a final step, we fit a spatiotemporal B-spline model to the discrete output at the finest scale to obtain a global, continuous representation of the velocity field.

$$
m_{p, q, r}\left(x_{0}, y_{0}, t_{0}\right)=\int_{\mathbb{R}^{3}} w\left(x-x_{0}, y-y_{0}, t-t_{0}\right)\left(x-x_{0}\right)^{p}\left(y-y_{0}\right)^{q}\left(t-t_{0}\right)^{r} f(x, y, t) d x d y d t
$$




\section{ROI TRACKING AND MOTION VISUALIZATION}

Since the motion is estimated for the complete sequence, the information of interest needs to be extracted and displayed in a proper way to facilitate the identification of wall motion abnormalities. Therefore, we compute radial velocity information from the estimated velocity field and visualize it inside a timevarying ROI. For each frame, the ROI is defined by a set of closed spline curves (typically, the inside and outside of the myocardium). For a given time $t$, each spline curve is parameterized as $\mathbf{r}_{t}(\tau)=\left(x_{t}(\tau), y_{t}(\tau)\right)$ in terms of the variable $\tau$. In particular, we represent $\mathbf{r}_{t}(\tau)$ as a linear combination of B-spline basis functions [14]

$$
\mathbf{r}_{t}(\tau)=\sum_{l=-\infty}^{\infty} \mathbf{c}_{t}(l) \beta^{n}\left(\frac{\tau}{m}-l\right)
$$

The integer $n$ denotes the degree of the B-spline and $\mathbf{c}_{t}(l)$ denotes a sequence of vector spline coefficients given by $\mathbf{c}_{t}(l)=\left(c_{t}(l), d_{t}(l)\right)$. The basis functions are dilated and shifted by some integer $m$ meaning that spline knots on the curve are located at distance $m$. Since the curves are closed, the sequence of spline coefficients $\mathbf{c}_{t}$ is periodic with some period length $K$. To track a curve in time, we compute a series of sample points $\mathbf{r}_{t}(k), k=0, \ldots, m K-1$, at integer distance on the curve. For these sample points, we compute the displacement from the continuous spline representation of the velocity field. By adding the displacements to the current position of the sample points, we obtain their position

$$
\hat{\mathbf{r}}_{t+1}(k)=\mathbf{r}_{t}(k)+\left(u\left(\mathbf{r}_{t}(k), t\right), v\left(\mathbf{r}_{t}(k), t\right)\right)
$$

in the next frame $t+1$, as shown in Fig. 2. To obtain a robust tracking, we approximate the $m K$ displaced sample points by the spline model (10) that has $K$ degrees of freedom, i.e., the spline coefficients $\mathbf{c}_{t+1}(l), l=0, \ldots, K-1$. These spline coefficients are determined by minimizing the least-squares approximation error

$$
\varepsilon^{2}=\sum_{k=0}^{m K-1}\left\|\mathbf{r}_{t+1}(k)-\hat{\mathbf{r}}_{t+1}(k)\right\|_{l_{2}}^{2}
$$

The choice of the parameter $m$ determines the tradeoff between closeness to the sample points and smoothness of the spline

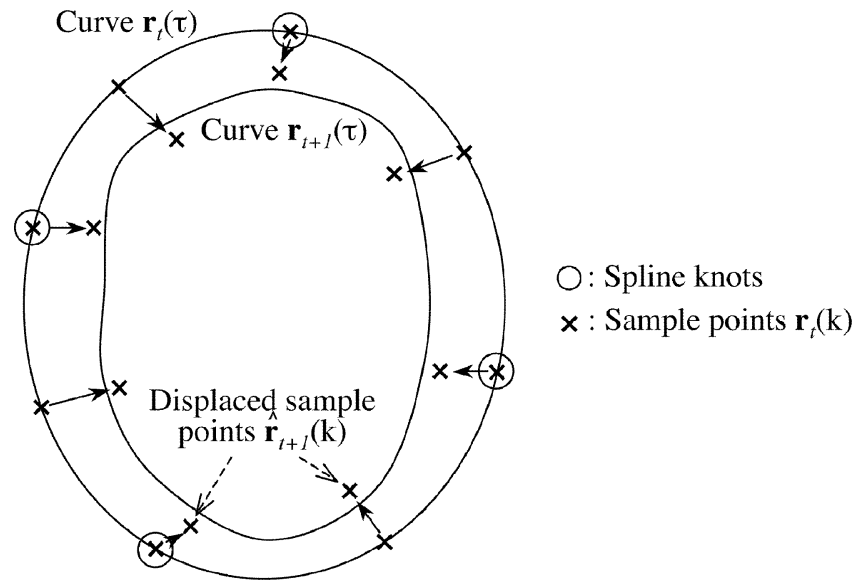

Fig. 2. ROI tracking by least-squares B-spline fitting to the estimated motion field.

curve. An efficient filter-based approach to compute the spline coefficients is described in [18], [19]. The set of obtained spline curves then defines the ROI at time $t+1$. To initialize the tracking process, the observer outlines the ROI by placing landmarks on the first frame of the image sequence. The user-defined landmarks are then used to obtain the initial spline curve. The corresponding spline coefficients $\mathbf{c}_{0}(l)$ in (10) are determined such that the curve exactly interpolates the landmarks $(m=1)$.

Having computed the ROI for each frame, we compute the centroids (centers of gravity), the areas, and the principal axes of inertia of the ROIs. These parameters can be computed exactly from the spline representation of the contours [20]. The velocity field of each frame is then projected onto the radial direction defined with respect to the centroid of the ROI of a particular frame (end diastolic or end systolic). Optionally, the velocity can also be projected onto the floating centroid of the ROI. In this case, the global translational motion of the ventricle is compensated by subtracting the velocity of the floating centroid from the estimated overall velocity. The choice of the optimal centroid (fixed versus floating) depends on the conditions under which the heart is imaged. Under conditions where excessive translation of the heart occurs, such as might be the case after cardiac surgery, a floating centroid approach would be more appropriate.

Inside the time-varying ROI, we color code the radial velocity using a similar color map as in tissue Doppler imaging (TDI). The color-coded velocity is then superimposed on the underlying image sequence in a semitransparent fashion. The color

$$
\begin{aligned}
& \mathbf{A}=\left(\begin{array}{cccccccc}
\left\langle w, I_{x}^{2}\right\rangle & \left\langle w, I_{x} I_{y}\right\rangle & \left\langle w, x I_{x}^{2}\right\rangle & \left\langle w, y I_{x}^{2}\right\rangle & \left\langle w, t I_{x}^{2}\right\rangle & \left\langle w, x I_{x} I_{y}\right\rangle & \left\langle w, y I_{x} I_{y}\right\rangle & \left\langle w, t I_{x} I_{y}\right\rangle \\
\cdot & \left\langle w, I_{y}^{2}\right\rangle & \left\langle w, x I_{x} I_{y}\right\rangle & \left\langle w, y I_{x} I_{y}\right\rangle & \left\langle w, t I_{x} I_{y}\right\rangle & \left\langle w, x I_{y}^{2}\right\rangle & \left\langle w, y I_{y}^{2}\right\rangle & \left\langle w, t I_{y}^{2}\right\rangle \\
\cdot & \cdot & \left\langle w, x^{2} I_{x}^{2}\right\rangle & \left\langle w, x y I_{x}^{2}\right\rangle & \left\langle w, x t I_{x}^{2}\right\rangle & \left\langle w, x^{2} I_{x} I_{y}\right\rangle & \left\langle w, x y I_{x} I_{y}\right\rangle & \left\langle w, x t I_{x} I_{y}\right\rangle \\
\cdot & \cdot & \cdot & \left\langle w, y^{2} I_{x}^{2}\right\rangle & \left\langle w, y t I_{x}^{2}\right\rangle & \left\langle w, x y I_{x} I_{y}\right\rangle & \left\langle w, y^{2} I_{x} I_{y}\right\rangle & \left\langle w, y t I_{x} I_{y}\right\rangle \\
& \cdot & \cdot & \cdot & \left\langle w, t^{2} I_{x}^{2}\right\rangle & \left\langle w, x t I_{x} I_{y}\right\rangle & \left\langle w, y t I_{x} I_{y}\right\rangle & \left\langle w, t^{2} I_{x} I_{y}\right\rangle \\
& \cdot & \cdot & \cdot & \left\langle w, x^{2} I_{y}^{2}\right\rangle & \left\langle w, x y I_{y}^{2}\right\rangle & \left\langle w, x t I_{y}^{2}\right\rangle \\
& \cdot & \cdot & \cdot & \cdot & \left\langle w, y^{2} I_{y}^{2}\right\rangle & \left\langle w, y t I_{y}^{2}\right\rangle \\
\mathbf{v} & \cdot & \cdot & \cdot & \cdot & \left\langle w, t^{2} I_{y}^{2}\right\rangle
\end{array}\right) \\
& \mathbf{b}=-\left(u_{0}, v_{0}, u_{x}, u_{y}, u_{t}, v_{x}, v_{y}, v_{t}\right)^{T}-\left(\left\langle w, I_{x} I_{t}\right\rangle,\left\langle w, I_{y} I_{t}\right\rangle,\left\langle w, x I_{x} I_{t}\right\rangle,\left\langle w, y I_{x} I_{t}\right\rangle,\left\langle w, t I_{x} I_{t}\right\rangle,\left\langle w, x I_{y} I_{t}\right\rangle,\left\langle w, y I_{y} I_{t}\right\rangle,\left\langle w, t I_{y} I_{t}\right\rangle\right)^{T} .
\end{aligned}
$$


display corresponds directly to myocardial contractility and allows to identify regions of abnormal motion more easily. The motion trajectory of the centroids, the principal axes of inertia and area-size of the ROIs can also be displayed. The movement of the ROI centroid allows to identify and quantify an underlying rigid translation of the myocardium. The motion of the principal axes of inertia allows to identify a global ventricular rotation (heart twisting).

To improve the contour tracking accuracy further, the proposed method may be combined with active contour techniques. However, most of these techniques are based on the detection of strong edges or ridges which do not necessarily correspond to the expert-defined ROI. Furthermore, the transition between the epicardial border and the surrounding tissue is usually very smooth and requires a more adapted definition of cardiac borders. The combination with trainable shape models may also improve the performance, but requires a large set of normalized training examples that also includes a variety of cases with abnormal motion. We did not consider these options here.

\section{NUMERICAL RESULTS}

For validation purposes, the algorithm was tested in three different ways. First, it was applied to synthetic data and compared to other motion estimation methods. Second, we analyzed echocardiograms of a rotating phantom. Third, the algorithm was applied to a set of clinical echocardiograms.

\section{A. Application to Synthetic Data}

A quantitative analysis of the performance of the algorithm is done on synthetic sequences for which the exact motion field is known.

1) Modeling of Echographic Texture: In order to simulate the appearance of clinical echo images, we use the linear image formation model of [21] and [22]. The model assumes that the echographic radio-frequency signal $R F(x, y)$ can be described by a spatial convolution between the system point spread function (PSF), $h(x, y)$, and the impulse response of the tissue $N(x, y)$

$$
R F(x, y)=\int_{\mathbb{R}^{2}} N(r, s) h(x-r, y-s) d r d s .
$$

The tissue $N(x, y)$ is modeled as a collection of point scatterers with 2-D normally distributed random echogenicity. The speed of sound throughout the tissue is assumed to be constant. The PSF is assumed to be space invariant with zero attenuation in the case of an adequately adjusted gain control. In particular, we use the Gabor function

$$
h(x, y)=\frac{1}{2 \pi \sigma_{x} \sigma_{y}} e^{i \omega_{0} x} e^{-(1 / 2)\left(\left(x^{2} / \sigma_{x}^{2}\right)+\left(y^{2} / \sigma_{y}^{2}\right)\right)}
$$

where $\sigma_{x}$ and $\sigma_{y}$ correspond to the axial and lateral dimension of the PSF, respectively. The parameter $\omega_{0}$ denotes the acoustic spatial frequency in axial direction. In order to simulate the radial propagation of the ultrasound beam, we divide the image plane into $K$ radial segments along the directions $\vartheta_{k}=k \pi / K$,

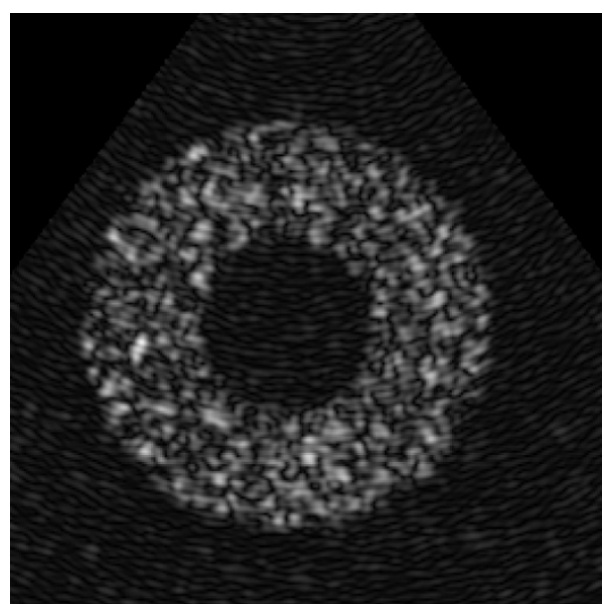

(a)

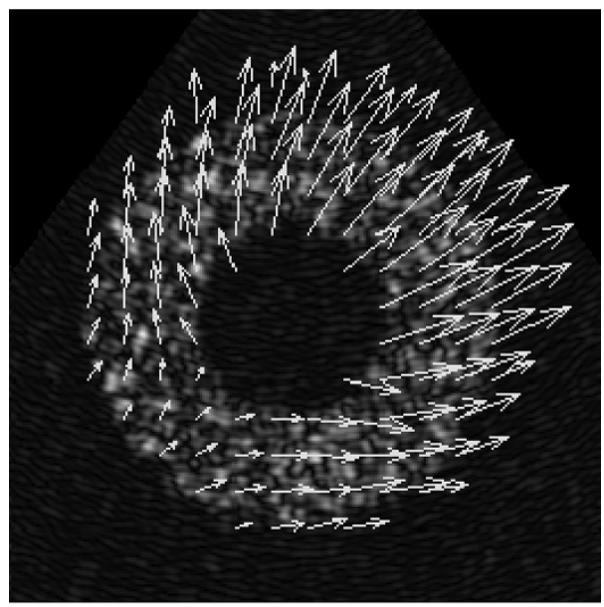

(b)

Fig. 3. (a) Frame of synthetic ultrasound sequence and (b) corresponding estimated velocity field.

$k=0, \ldots, K-1$. The oriented filter $h\left(x^{\prime}, y^{\prime}\right)$ in each segment is obtained from the basic filter via the coordinate transformation

$$
\begin{aligned}
& x^{\prime}=\cos \left(\vartheta_{k}\right) x+\sin \left(\vartheta_{k}\right) y \\
& y^{\prime}=-\sin \left(\vartheta_{k}\right) x+\cos \left(\vartheta_{k}\right) y
\end{aligned}
$$

The resulting B-mode images are given by the modulus (envelope) of the complex echographic radio-frequency signal. The B-mode image gray level histogram has a Rayleigh distribution which is also known as "fully formed" speckle. In this experiment, we have used the parameter set $\sigma_{x}=2.0$ pixels, $\sigma_{y}=2.8$ pixels, $\omega_{0}=\pi / 2$, and $K=180$.

2) Modeling of Heart Motion: In this study, we simulate an apical short-axis view of a heart. For simplicity, we consider a circular heart model and simulated a periodic displacement field that maintains the area of the myocardium constant. This is achieved by applying a radial displacement field with a magnitude decreasing with the distance from the center. This displacement field is cosine modulated in time to simulate the expansion and contraction. This kind of motion reflects the wall thinning and thickening of a beating heart during diastole and systole. Additional to the myocardial excursion, 


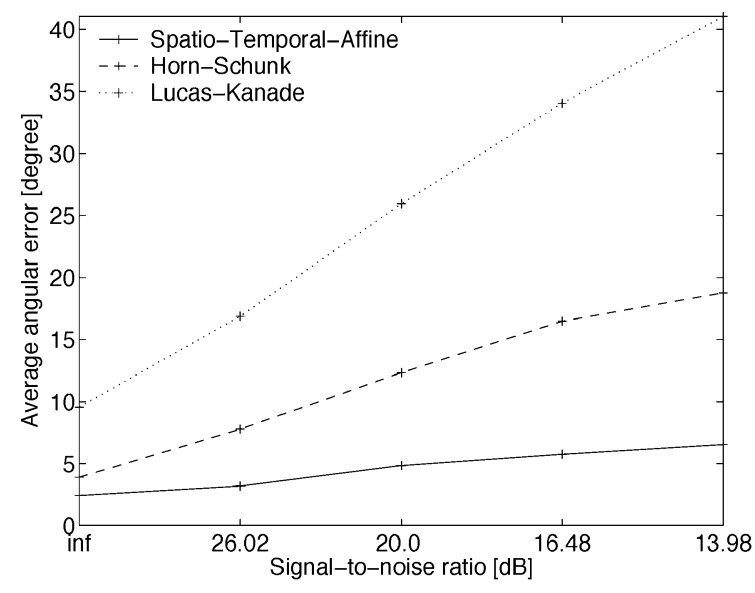

(a)

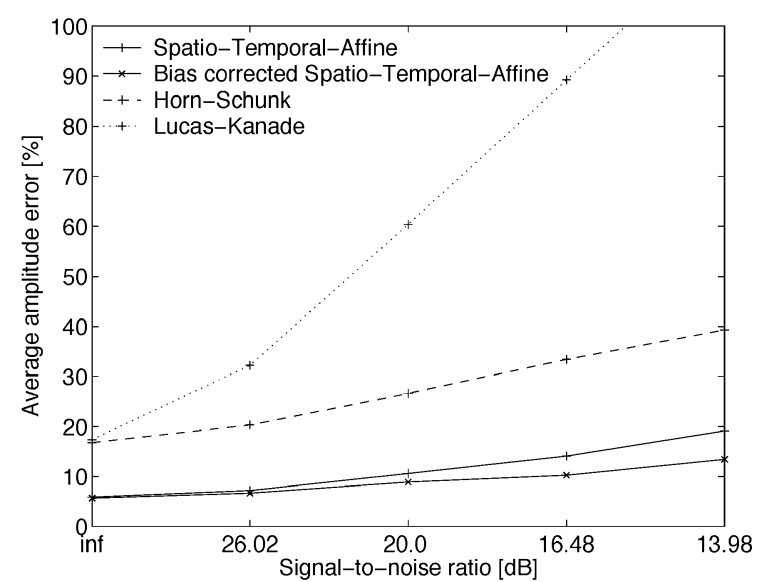

(b)

Fig. 4. Average angular and amplitude errors of different methods for different SNRs of the point-scatterer images. (a) Average angular error. (b) Average amplitude error.

the heart model is translated toward the upper-right direction to simulate an underlying rigid motion. The known motion field is applied to the initial point scatterer image to obtain a sequence of warped images. The dynamic model was set such that the maximum velocity per frame is 2.0 pixels. In order to simulate additional changes of the scatterer echogenicity that are not caused by motion, we added normally distributed zero-mean white noise to each frame of the warped image sequence. This models, for example, echogenicity changes that are caused by blood flow. This is a simplified, not entirely realistic noise model, but allows us to test the robustness of the algorithms. The final echo sequence is computed by applying the image formation process described above to each frame of the perturbed point scatterer images. As an example, one frame of a 32-frame test sequence is shown in Fig. 3(a). In this case, the signal-to-noise ratio (SNR) of the point-scatterer images is $13.98 \mathrm{~dB}$.

3) Motion Estimation Results: The applied motion field was estimated by the algorithm from the simulated B-mode images. As an example, the corresponding estimated velocity field of Fig. 3(a) is shown in Fig. 3(b). The performance of the algorithm was tested on sequences that were obtained from point scatterer images of different SNRs. The method was compared with the Lucas-Kanade [11] and the Horn-Schunk [9] optical flow methods. For the Lucas-Kanade method, we have used the same parameter set as proposed in [23]. For the Horn-Schunk method, we performed, at most, 250 steps of the iterative algorithm and used a range of regularization parameters $1 \leq$ $\alpha \leq 150$. All sequences were prefiltered in space with a $\mathrm{Bi}-$ nomial filter of variance $\sigma_{s}^{2}=1.0$. The experiments were performed on a 400-MHz Macintosh G4 computer. The computation time of the proposed algorithm applied to the test sequences [32 (256 × 256)-frames] was $44 \mathrm{~s}$. To assess the performance of these algorithms, we used two error measures. The first is the angular error between the estimated velocity $\widehat{v}$ and the exact velocity $\mathbf{v}$ which is given by

$$
\theta=\arccos \frac{\langle\mathbf{v}, \widehat{\mathbf{v}}\rangle}{\|\mathbf{v}\|_{l_{2}}\|\widehat{\mathbf{v}}\|_{l_{2}}}
$$

TABLE II

ERror MEASURes of DifFERENT Optical Flow Methods

\begin{tabular}{c||c|c}
\hline Method & $\begin{array}{c}\text { Average angular } \\
\text { error } \bar{\theta}\left[{ }^{\circ}\right]\end{array}$ & $\begin{array}{c}\text { Average amplitude } \\
\text { error } \bar{\varepsilon}[\%]\end{array}$ \\
\hline \hline Lucas-Kanade & $9.55 \pm 16.93$ & $17.4 \pm 73.8$ \\
\hline Horn-Schunk & & \\
$\alpha=15$ & $7.77 \pm 13.86$ & $20.3 \pm 59.8$ \\
$\alpha=75$ & $3.90 \pm 7.03$ & $16.81 \pm 21.6$ \\
$\alpha=135$ & $4.05 \pm 5.92$ & $29.6 \pm 18.1$ \\
\hline Spatio-Temporal-Affine & $2.43 \pm 6.10$ & $5.9 \pm 15.2$ \\
\hline
\end{tabular}

This error measure does not depend on the magnitude of the motion vectors but describes the directional error of the motion vectors. The second is the relative error of the velocity magnitude which is calculated as

$$
\varepsilon=\frac{\left|\|\mathbf{v}\|_{l_{2}}-\|\widehat{\mathbf{v}}\|_{l_{2}}\right|}{\|\mathbf{v}\|_{l_{2}}} .
$$

The mean errors $\bar{\theta}$ and $\bar{\varepsilon}$ and their corresponding standard deviations were computed by averaging over all pixels in the image sequence.

To test the accuracy of the different methods, they were first applied to echo sequences that were obtained from point scatterer images without additional noise. The resulting errors are summarized in Table II. The worst method is the Lucas-Kanade method. This is due to the fact that the diverging character of the motion field is not compatible with the local constancy assumption and that it works only at a single scale. The method of Horn-Schunk performs better with respect to the angular error measure. Table II shows the lowest possible angular error for the Horn-Schunk method that was obtained for the regularization parameter $\alpha=75$. However, the regularization parameter depends on the image sequence and the best choice is not known a priori. Moreover, an increase of the regularization parameter leads to biased velocity estimates toward lower values which is reflected by the relatively high-amplitude error $\bar{\varepsilon}=29.6 \% \pm 18.1 \%$ for $\alpha=135$. Low values for $\alpha$ reduce the 
bias but lead to an increased directional error. The spatiotemporal-affine method performs best since it is well adapted to the underlying motion pattern.

The robustness of the algorithms in the presence of additional scatterer noise is illustrated in Fig. 4. The algorithms were applied to echo sequences that were obtained from point scatterer images of SNRs varying from 26.02 to $13.98 \mathrm{~dB}$. Fig. 4(a) shows the average angular errors of the three methods. For each noise level, we have chosen the regularization parameter of the Horn-Schunk method such that the error was minimal. The corresponding amplitude errors are illustrated in Fig. 4(b). The Lucas-Kanade method is the most sensitive one, whereas the Horn-Schunk method performs reasonably well if the regularization parameter is chosen properly. The spatiotemporal-affine algorithm performs best with respect to both error measures because the multiresolution strategy and the use of a temporal window provide increased robustness against noise. However, it can be shown in general that the least-squares method, as used in (9), yields estimates that are biased toward smaller values if the data matrix contains noise [24]. This can also be observed from the experiments by computing the relative bias

$$
\varepsilon_{b}=\frac{\|\mathbf{v}\|_{l_{2}}-\|\widehat{\mathbf{v}}\|_{l_{2}}}{\|\mathbf{v}\|_{l_{2}}}
$$

The mean bias $\overline{\varepsilon_{b}}$, averaged over the whole image sequence, was calculated for each noise level. The underestimation grows with increasing noise level and ranged from $1.7 \%$ to $11.4 \%$. To demonstrate the influence of the bias to the amplitude error (18), we recalculated it after correcting the velocity estimates by the average bias such that

$$
\widehat{\mathbf{v}}=\frac{1}{1-\overline{\varepsilon_{b}}} \widehat{\mathbf{v}} .
$$

The resulting amplitude error is also plotted in Fig. 4(b); it is smaller than in the noncorrected case, confirming our observation. A more systematic way to correct for the bias is given by the total least-squares (TLS) technique [24], [25]. However, this technique requires the knowledge of the noise covariance of a system matrix that is composed by the original data matrix and the right hand side vector. Its estimation is challenging if the sample sizes are small and if an accurate noise model is not known. Our experiments with the TLS method (not reported here) were not satisfactory. Of all the techniques we tried, the proposed least-squares fit of the spatiotemporal-affine motion model in combination with the bias correction (20) performed best.

The experiments demonstrate the robustness of the proposed algorithm in the case of a simple ultrasound imaging model. More realistic simulations should, perhaps, consider a nonlinear model for the image formation and a tissue model that reflects the heart anatomy, such as fiber directions and the nonuniformity of the speed of sound, more closely. However, these properties are difficult to simulate which calls for alternative validation methods, such as phantom experiments.

4) Radial Motion Visualization: For radial motion visualization, the estimated velocity was projected onto the direction

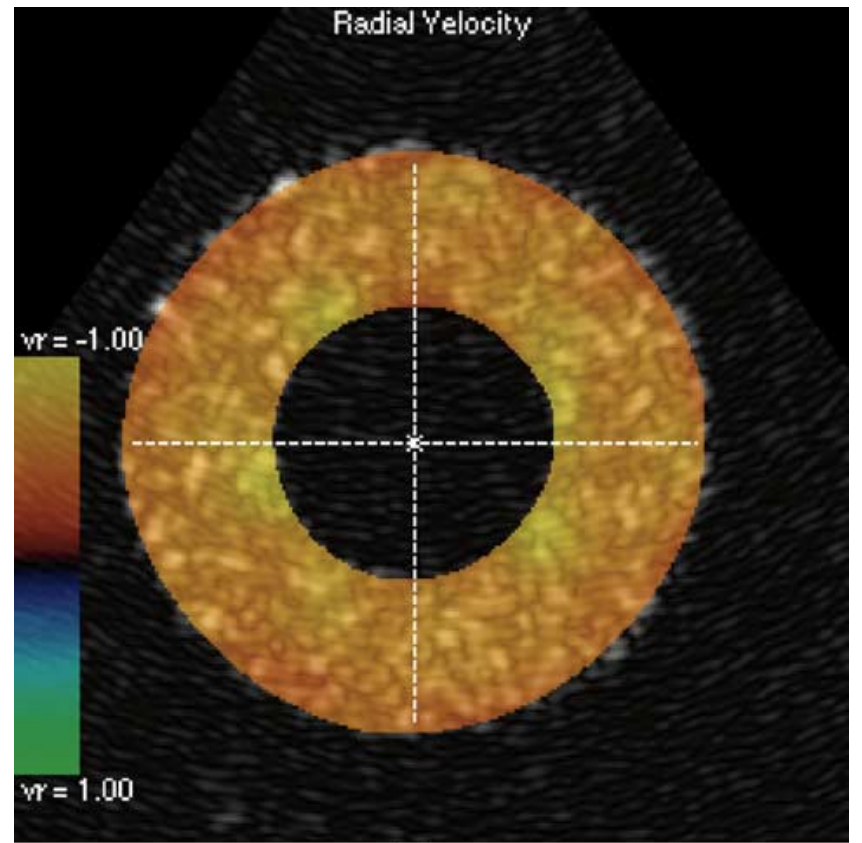

(a)

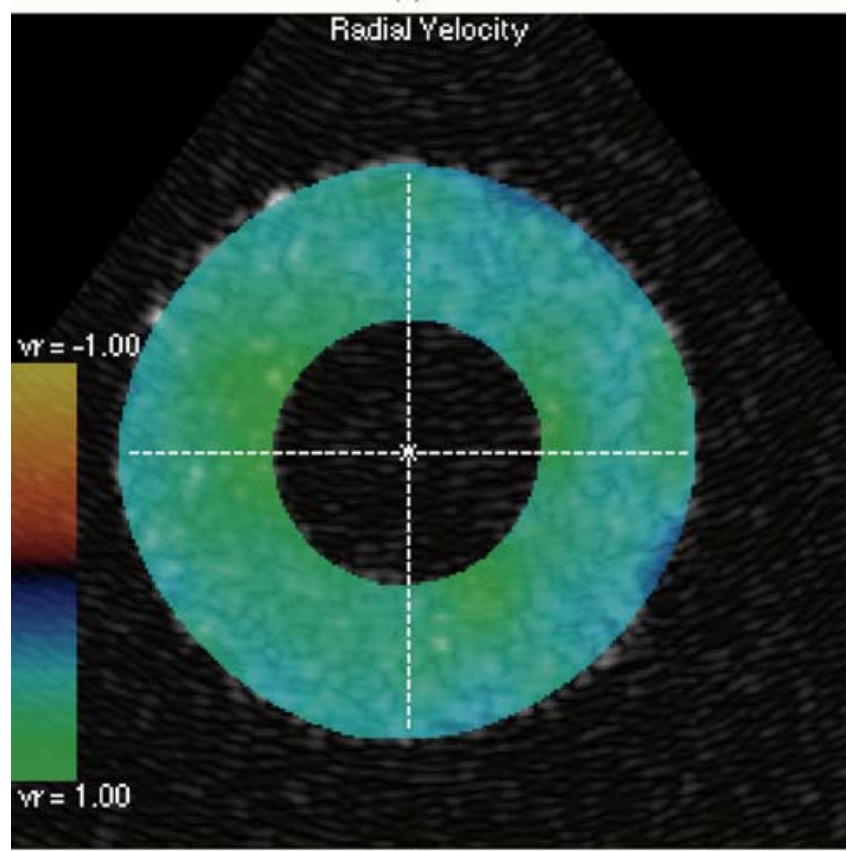

(b)

Fig. 5. Synthetic ultrasound sequence and superimposed color-coded radial velocity. (a) Radial velocity during systole. (b) Radial velocity during diastole.

to the floating centroid of the ROI to compensate for the underlying translational motion. The color-coded radial velocities for the example sequence are shown in Fig. 5(a) and (b) for systole and diastole, respectively. The uniform contraction during systole is clearly expressed by the red/yellow colors. On the other hand, the expansion during diastole is correctly displayed by the blue/green colors. The color display is more intuitive than the velocity field representation and is not distorted by the underlying global translation. The ROI also clearly tracks the myocardium and the circular form of the ROI contours is maintained due to the least-squares fitting to the estimated noisy motion field. 


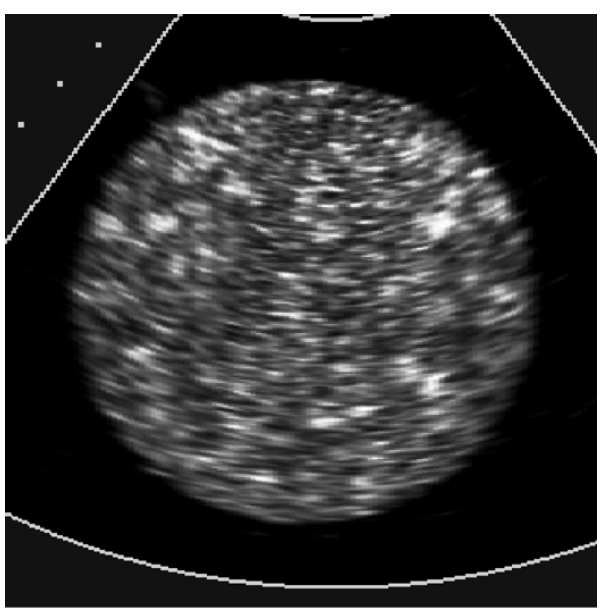

(a)

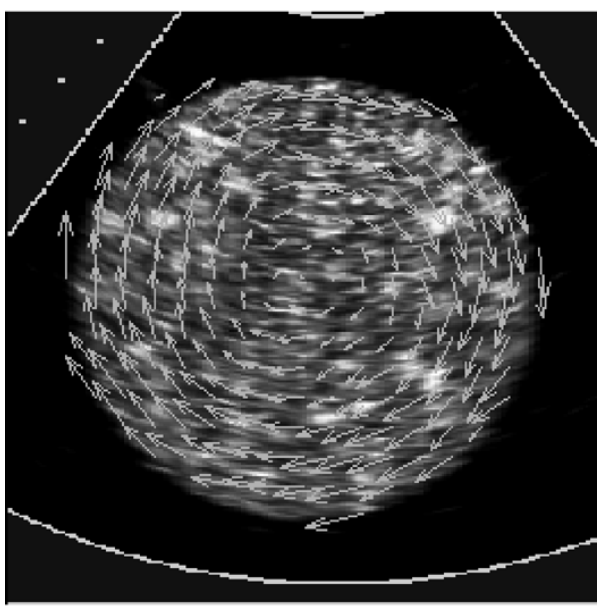

(b)

Fig. 6. (a) One frame of the rotating phantom sequence and (b) its corresponding estimated velocity field

\section{B. Phantom Study}

The algorithm's ability to estimate motion from real echocardiograms was tested in a phantom experiment. A cylinder-shaped, tissue-mimicking phantom was placed inside a tube of water and rotated with constant angular velocity around its longitudinal axis. The gelatine phantom had a diameter of $7.5 \mathrm{~cm}$ and was made out of a $2 \%$ agar-agar solution containing randomly dispersed scatterers ranging from 50 to $250 \mu \mathrm{m}$. Echocardiograms of a 2-D cross section orthogonal to the axis of rotation were acquired and analyzed by the algorithm. Fig. 6(a) shows one frame of a rotating phantom echocardiogram and Fig. 6(b) shows its corresponding estimated velocity field. Since the applied motion is purely rotational, the velocity magnitude increases linearly with the radius. To test this relationship for the estimated velocity field, we computed local means and standard deviations of the velocity field along annuli of different radii. We then fitted a linear regression line to these mean velocities as shown in Fig. 7. The coefficient of determination is 0.993 which confirms the linear relationship. The slope of the regression line corresponds to the estimated angular velocity. The estimated value was $0.0727 \mathrm{rps}$, whereas the true angular velocity applied to the phantom was

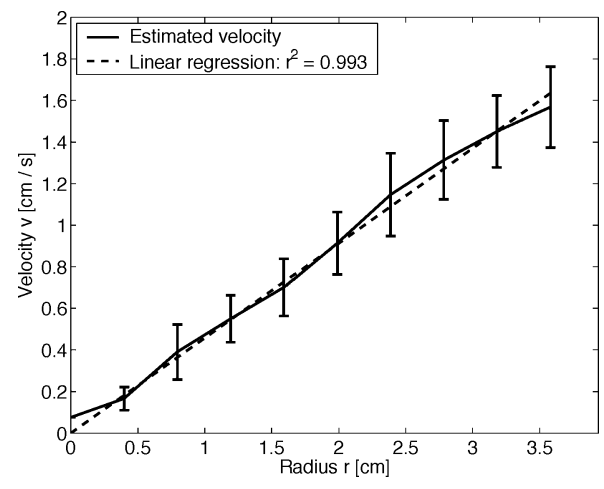

Fig. 7. Means and standard deviations of the estimated velocity field for fixed radii are plotted together with its linear regression line.
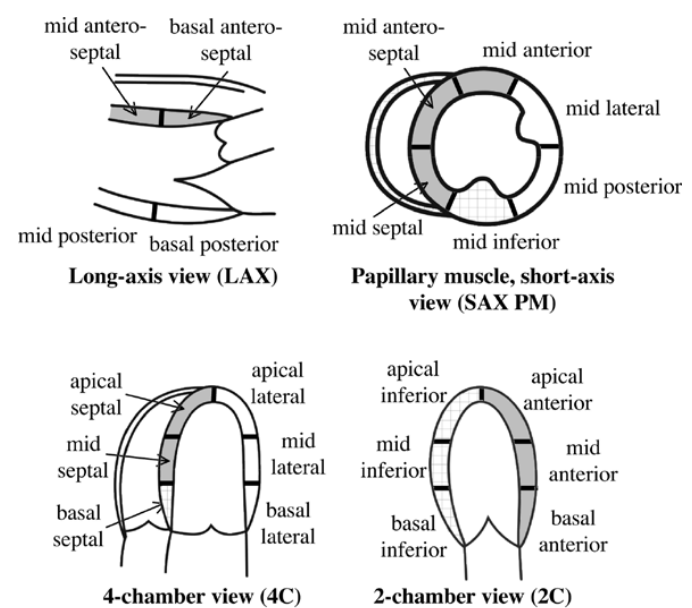

$$
\begin{aligned}
& \square \text { Left anterior descending coronary artery (LAD) } \\
& \square \text { Left circumflex coronary artery (LCX) } \\
& \square \text { Right coronary artery (RCA) }
\end{aligned}
$$

Fig. 8. Standard definition of left ventricular segments for 2-D echocardiography. Regions of blood supply by the different coronary arteries are marked accordingly.

$0.0796 \mathrm{rps}$. This corresponds to an underestimation of $8.7 \%$. This experiment can also serve as a calibration tool to determine the factor for the bias correction of the motion estimates as proposed in (20).

\section{Application to Clinical Data}

For a first in vivo validation, we applied the method to a set of clinical echocardiograms that were obtained from an animal study. We analyzed ultrasound sequences of six dogs with infarctions that were artificially induced by the occlusion of the left anterior descending artery (LAD). Fig. 8 shows a standardized division of the left ventricle into 16 segments according to the American Heart Association [26]. Regions that depend on the left anterior descending artery blood supply are also indicated. Echocardiograms were acquired in an open-chest state before and after the infarction. Since the infarction was caused manually, the exact localization and extend is known and a direct comparison between normal and pathological cases is possible. For one case, two frames of a long-axis view (LAX) before the infarction are shown in Fig. 9(a) and (c) for systole and 


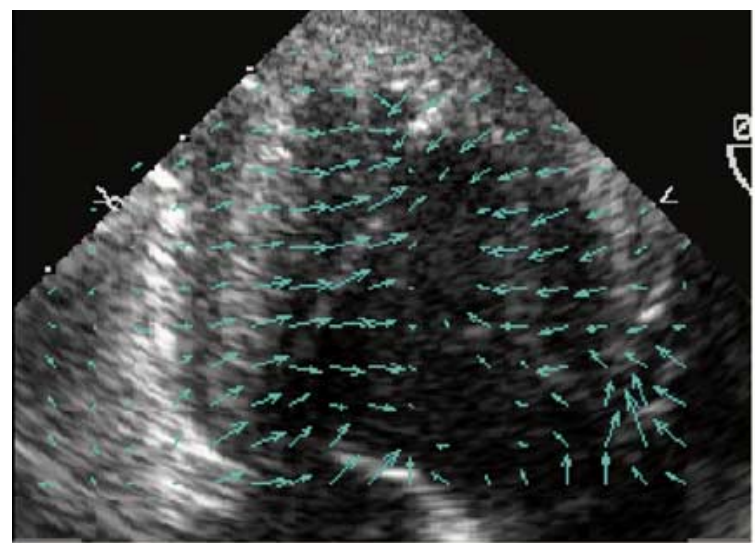

(a)

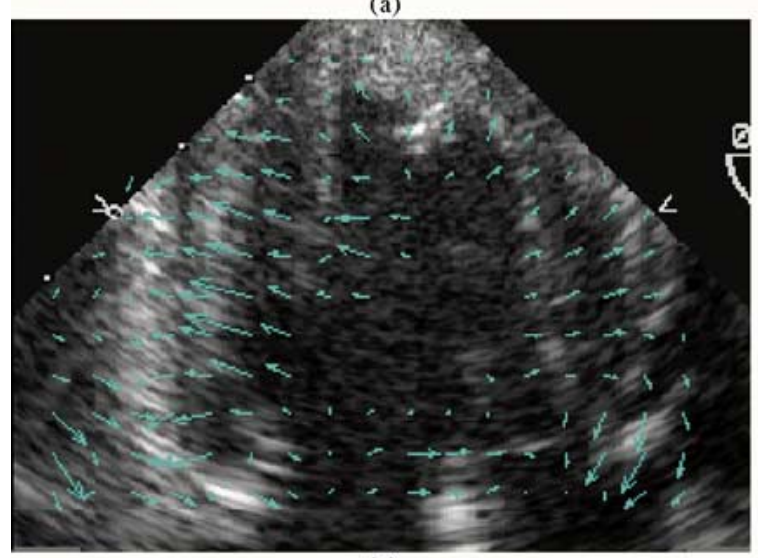

(c)

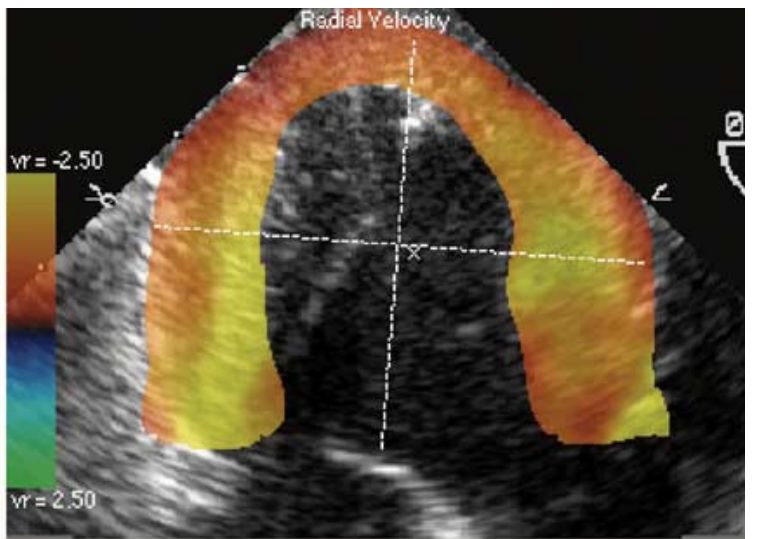

(b)

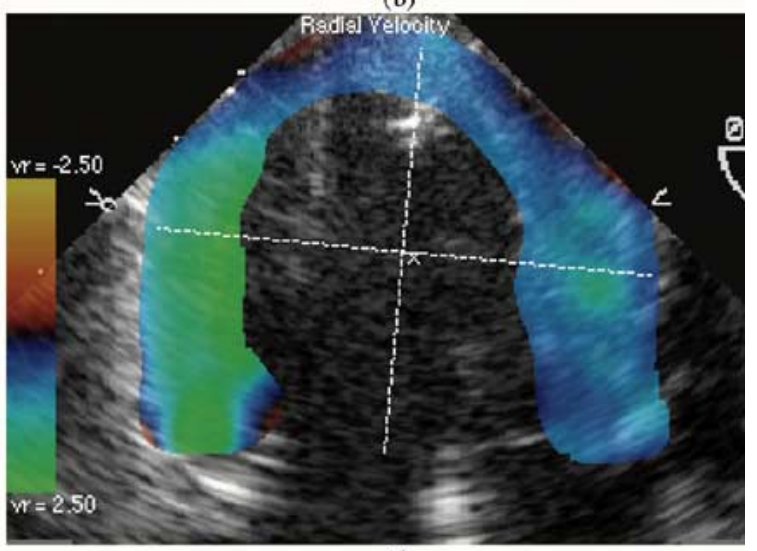

(d)

Fig. 9. Echocardiograms with superimposed motion information before infarction. The velocity fields and colors reflect the normal contraction and expansion during systole and diastole. (a) Estimated velocity field during systole. (b) Color-coded radial velocity during systole. (c) Velocity field during diastole. (d) Color-coded radial velocity during diastole. The principal axes of inertia of the ROI are indicated by dotted lines.

diastole, respectively. The corresponding motion estimation results are superimposed in the form of a needle diagram. The typical ventricular contraction and expansion of a normal beating heart during systole and diastole is clearly captured by the estimated motion fields. Fig. 10 shows the status after the infarction. Fig. 10(a) and (c) correspond to a frame during systole and diastole, respectively. In both figures, the dyskinesia (paradoxical motion) in the apical to mid anteroseptal segments (upper-right region) is correctly captured by the motion field; during systole, the affected segments move outwards due to the inner blood pressure, whereas they move inwards during diastole.

The color-coded display of the radial velocity confirms the observations obtained from the motion fields, but can be interpreted in a more straightforward way. The color-coded radial velocity of the example case before the infarction is shown in Fig. 9. Fig. 9(b) and (d) show the corresponding frames during systole and diastole, respectively. In all cases, the velocity was projected onto a fixed reference point that is given by the ROI centroid at end diastole. The regular contraction of the whole myocardium during systole is indicated by the red/yellow colors, whereas the blue/green colors reflect the normal expansion during diastole. The color-coded radial velocity after the infarction is shown in Fig. 10. In Fig. 10(b), the outward motion of the apical to mid anteroseptal segments (upper-right region) during systole is correctly visualized by the blue/green colors. The infarction also causes an outward movement of the basal posterior (lower left) segment. Fig. 10(d) shows the pathological motion during diastole. Here, the red/yellow colors indicate that the apical to mid anteroseptal segments (upper-right region) move inwards instead of outwards as in the normal case. Accordingly, the basal posterior (lower left) segment also moves inwards.

1) Segmental Wall Motion Analysis: For all six cases, we compared peak radial velocities of myocardial segments during systole before and after the infarction. In particular, we analyzed segments that were classified as dyskinetic by the expert. Since, during systole, the ventricle is contracting (negative radial velocity), a normal beating heart does not exhibit significant positive radial velocities. However, dyskinetic segments expand during systole and show significant positive values. In this experiment, eight segments out of a total of 36 were classified as dyskinetic by the expert. The average of the measured positive peak radial velocities in these dyskinetic segments is $1.99 \pm$ $0.92 \mathrm{~cm} / \mathrm{s}$. In contrast, the average peak velocity in these segments before the infarction was $0.18 \pm 0.15 \mathrm{~cm} / \mathrm{s}$. A paired t-test shows a significant difference between the two states $(p=$ 0.000 ). Although differences are clearly significant for the dyskinetic case, the distinction between normal, akinetic (no motion), and hypokinetic (very little motion) cases is not so well defined. Statistical evidence in these cases could not be established since the experiment only included six hearts that also show a variation in velocities before the infarction. To perform 


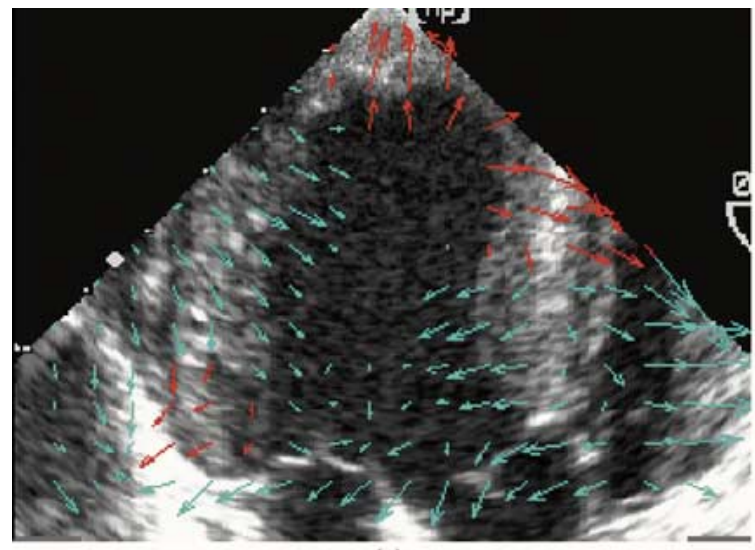

(a)

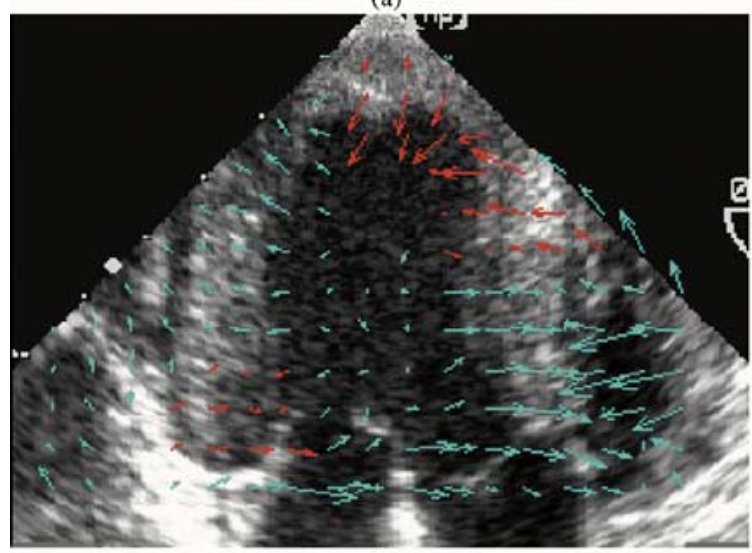

(c)

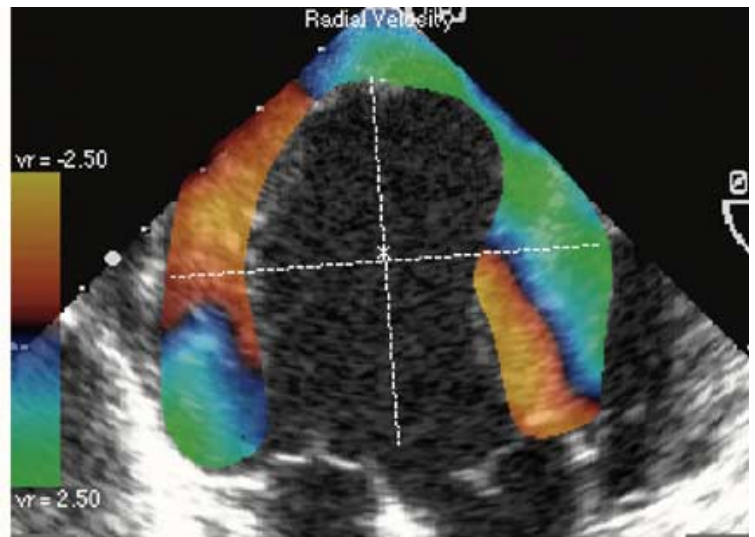

(b)

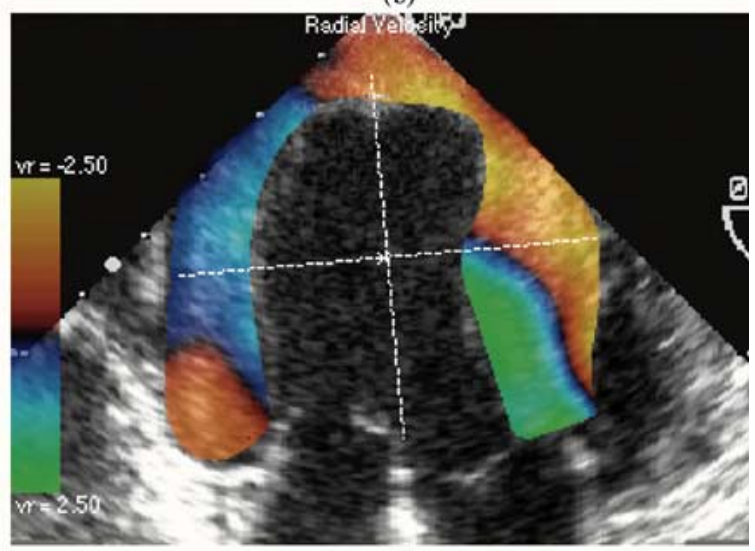

(d)

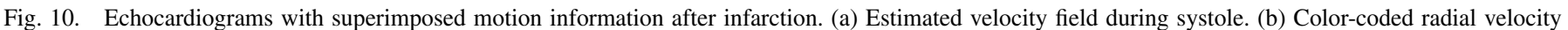

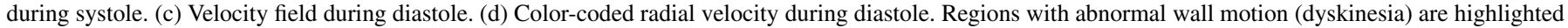
by red arrows. The principal axes of inertia of the ROI are indicated by dotted lines.

a meaningful statistical study with a higher number of infarct categories, a larger number of cases needs to be analyzed.

2) ROI Tracking Validation: The motion estimation algorithm in combination with the automatic contour tracking was also validated by comparing it to the manual contour tracking by an expert. Since the epicardial border is, in general, not clearly defined and is only partially imaged in our test sequences, the validation is performed by tracking the endocardial border of the left ventricle. In all 12 test sequences, an expert, who was blind to the computer analysis, outlined the contours of the endocardial border at the end-diastolic and end-systolic frames, respectively. Each contour was defined by a set of landmarks that were interpolated by a cubic B-spline curve. In this experiment, the curves were not closed, but the two landmarks at the mitral valve attachment were connected by a straight line to define an endocardial area. The manually defined B-spline contour at end diastole was then tracked by the algorithm to end systole. We calibrated the system and used a constant bias correction factor in all experiments. Fig. 11(a) and (c) show one example of the manually determined contours (green line) at end diastole and end systole, respectively. The automatically tracked contour is shown in Fig. 11(b) and (c) for mid systole and end systole, respectively (white line). The areas that are enclosed by the manually determined contours at end diastole and end systole are denoted by the characteristic functions $\Omega_{E D}$ and $\Omega_{E S}$, respectively. The area defined by the automatically tracked contour at end systole is denoted as $\widehat{\Omega}_{E S}$. By calculating the area size of a characteristic function as

$$
\|\Omega\|_{L_{1}}=\int_{\mathbb{R}^{2}}|\Omega(x, y)| d x d y
$$

we define the manually determined area ejection fraction as $E F=\left(\left\|\Omega_{E D}\right\|_{L_{1}}-\left\|\Omega_{E S}\right\|_{L_{1}}\right) /\left\|\Omega_{E D}\right\|_{L_{1}}$. Accordingly, we denote the computer-based area ejection fraction by $\widehat{E F}=$ $\left(\left\|\Omega_{E D}\right\|_{L_{1}}-\left\|\widehat{\Omega}_{E S}\right\|_{L_{1}}\right) /\left\|\Omega_{E D}\right\|_{L_{1}}$. The area ejection fraction can be interpreted as the 2-D analog of the volumetric ejection fraction [27] and relates the endocardial area before and after the contraction phase (systole). To compare the automatically tracked contours with the expert standard, we compute two error measures. The first is the relative ejection fraction error $E_{E F}=|E F-\widehat{E F}| /|E F|$. Let $\left\|\Omega_{E S}-\widehat{\Omega}_{E S}\right\|_{L_{1}}$ denote the area size of the characteristic function that describes the region where the end-systolic manual and computer-based masks do not coincide. Then, the second error measure $E_{A}=$ $\left\|\Omega_{E S}-\widehat{\Omega}_{E S}\right\|_{L_{1}} /\left\|\Omega_{E S}\right\|_{L_{1}}$ corresponds to the relative size of the area, where the manual and computer-defined masks do not overlap. In this experiment, the average area error was determined as $E_{A}=10.20 \pm 2.04 \%$ and the area-ejection fraction error was $E_{E F}=11.42 \pm 7.26 \%$. To determine the variability of the expert contour tracking, the contours at end systole were outlined a second time by the observer. The same error 


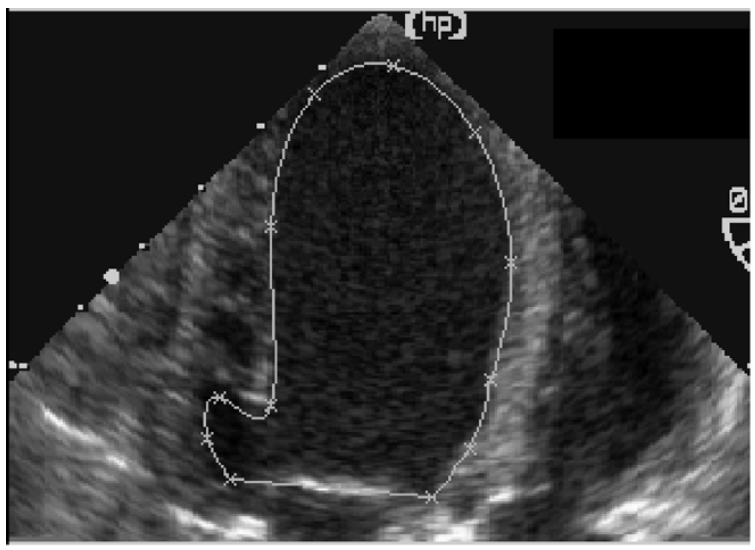

(a)

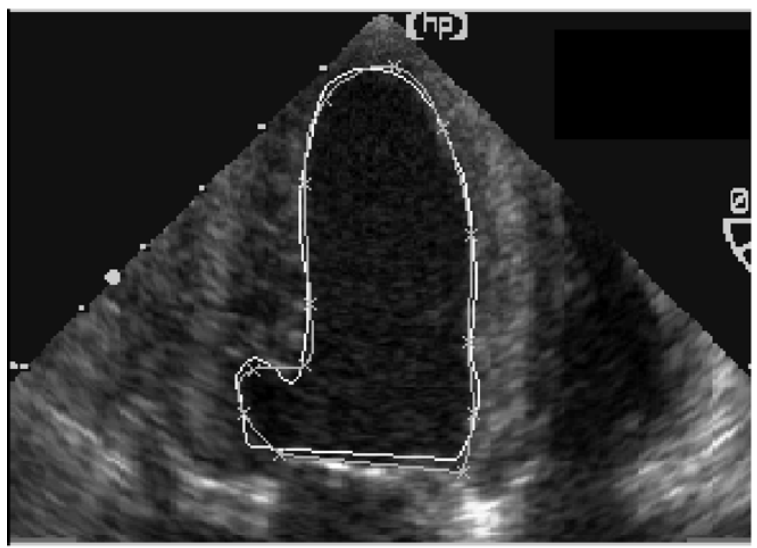

(c)

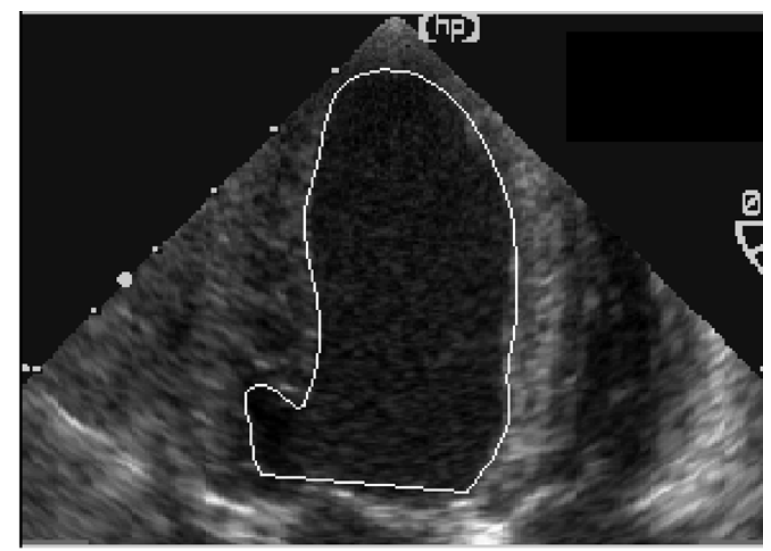

(b)

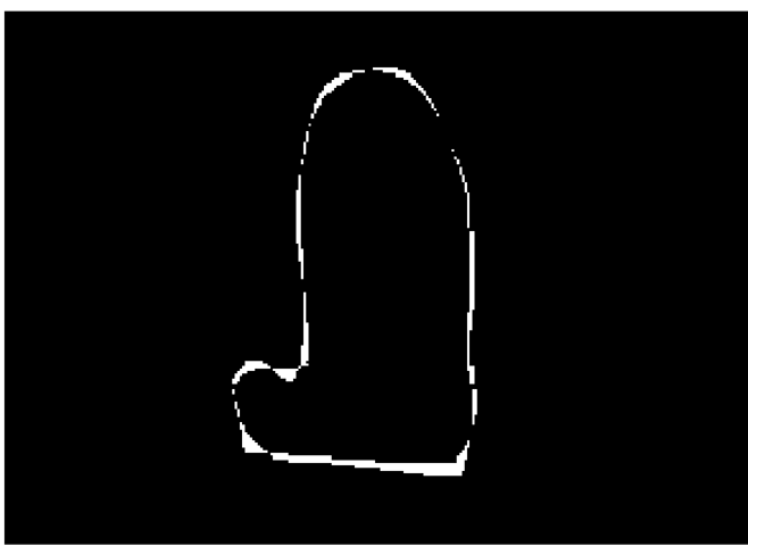

(d)

Fig. 11. Comparison between manual and automatic contour tracking. The initial manually defined contour at end diastole is automatically tracked to end systole and compared with the manual result. (a) Manually defined contour at end diastole. (b) Automatically tracked contour at mid systole. (c) Manually defined contour (defined by landmarks) and automatically tracked contour at end systole. (d) Endocardial area at end systole for which the manual and computer-based masks do not coincide.

measures as in the manual/computer comparison were calculated. The intra-observer error for the end-systolic area was determined as $E_{A}=10.56 \pm 3.45 \%$ and the intra-observer area ejection fraction error was $E_{E F}=13.62 \pm 9.86 \%$. These results show that the motion estimation algorithm yields realistic results and that the contours are sufficiently well tracked to provide a time-varying ROI for further post processing and motion visualization.

\section{CONCLUSION}

We proposed a new method to estimate heart motion from echocardiograms that uses a local motion model that is particularly well adapted to typical heart dynamics. The method is fast and robust due to a wavelet-like multiresolution implementation. For visualization purposes, we developed a robust method to track a ROI in time. Inside the time-varying ROI, we superimpose a color-coded radial velocity component onto the echocardiogram that allows to directly identify and quantify myocardial contractility. The algorithm outperforms other methods on simulated data; its applicability to real echocardiograms was demonstrated by a phantom experiment. A first validation of the proposed method on clinical echocardiograms yielded realistic motion fields. We also verified that the estimated regional wall motion was in good agreement with the expert echocardiographic reading.

\section{REFERENCES}

[1] P. R. Hunziker, M. H. Picard, N. Jander, M. Scherrer-Crosbie, M Pfisterer, and P. T. Buser, "Regional wall motion assessment in stress echocardiography by tissue Doppler bull's-eyes," J. Amer. Soc. Echocardiograph., vol. 12, no. 3, pp. 196-202, Mar. 1999.

[2] A. E. Weyman, Principles and Practice of Echocardiography, 2nd ed. Philadelphia, PA: Lea \& Febiger, 1994.

[3] M. Sonka and J. Fitzpatrick, Eds., Handbook of Medical Imaging. Bellingham, WA: SPIE, 2000, vol. 2, Cardiac Image Analysis: Motion and Deformation, ch. 12.

[4] M. Kass, A. Witkin, and D. Terzopoulos, "Snakes: Active contour models," Int. J. Comput. Vis., vol. 1, pp. 321-332, Jan. 1988.

[5] V. Chalana, D. Linker, D. Haynor, and Y. Kim, "A multiple active contour model for cardiac boundary detection on echocardiographic sequences," IEEE Trans. Med. Imag., vol. 15, no. 6, pp. 290-298, Jun. 1996.

[6] G. Jacob, J. Noble, C. Behrenbruch, A. Kelion, and A. Banning, "A shape-space-based approach to tracking myocardial borders and quantifying regional left-ventricular function applied in echocardiography," IEEE Trans. Med. Imag., vol. 21, no. 3, pp. 226-238, Mar. 2002.

[7] J. Bosch, S. Mitchell, B. Lelieveldt, F. Nijland, O. Kamp, M. Sonka, and J. Reiber, "Automatic segmentation of echocardiographic sequences by active appearance motion models," IEEE Trans. Med. Imag., vol. 21, no. 11, pp. 1374-1383, Nov. 2002.

[8] G. E. Mailloux, F. Langlois, P. Y. Simard, and M. Bertrand, "Restoration of the velocity field of the heart from two-dimensional echocardiograms," IEEE Trans. Med. Imag., vol. MI-8, no. 6, pp. 143-153, Jun. 1989. 
[9] B. Horn and B. Schunk, "Determining optical flow," Artif. Intell., vol. 17, pp. 185-203, 1981.

[10] G. Zini, A. Sarti, and C. Lamberti, "Application of continuum theory and multi-grid methods to motion evaluation from 3D echocardiography," IEEE Trans. Ultrason., Ferroelect., Freq. Contr., vol. 44, no. 3, pp. 297-308, Mar. 1997.

[11] B. Lucas and T. Kanade, "An iterative image restoration technique with an application to stereo vision," in Proc. DARPA IU Workshop, 1981, pp. 121-130.

[12] Y. Chunke, K. Terada, and S. Oe, "Motion analysis of echocardiograph using optical flow method," in Proc. IEEE Int. Conf. Systems, Man, and Cybernetics, vol. 1, Oct. 1996, pp. 672-677.

[13] P. Baraldi, A. Sarti, C. Lamberti, A. Prandini, and F. Sgallari, "Evaluation of differential optical flow techniques on synthesized echo images," IEEE Trans. Biomed. Eng., vol. 43, no. 3, pp. 259-272, Mar. 1996.

[14] M. Unser, "Splines: A perfect fit for signal and image processing," IEEE Signal Process. Mag., vol. 16, no. 6, pp. 22-38, Nov. 1999.

[15] X. Zong, A. Laine, and E. Geiser, "Speckle reduction and contrast enhancement of echocardiograms via multiscale nonlinear processing," IEEE Trans. Med. Imag., vol. 17, no. 8, pp. 532-540, Aug. 1998.

[16] S. Mallat, "A theory for multiresolution signal decomposition: The wavelet representation," IEEE Trans. Pattern Anal. Mach. Intell., vol. 11, no. 7, pp. 674-693, Jul. 1989.

[17] M. Sühling, M. Arigovindan, P. Hunziker, and M. Unser, "Multiresolution moment filters: Theory and applications," IEEE Trans. Image Process., vol. 13, no. 4, pp. 484-495, Apr. 2004.

[18] M. Unser, A. Aldroubi, and M. Eden, "B-Spline signal processing: Part I - Theory," IEEE Trans. Signal Process., vol. 41, no. 2, pp. 821-833, Feb. 1993.

[19] — - "B-Spline signal processing: Part II — Efficient design and applications," IEEE Trans. Signal Process., vol. 41, no. 2, pp. 834-848, Feb. 1993.

[20] M. Jacob, T. Blu, and M. Unser, "An exact method for computing the area moments of wavelet and spline curves," IEEE Trans. Pattern Anal. Mach. Intell., vol. 23, no. 6, pp. 633-642, Jun. 2001.

[21] J. C. Bamber and R. J. Dickinson, "Ultrasonic b-scanning: A computer simulation," Phys. Med. Biol., vol. 25, pp. 463-479, 1980.

[22] M. Bertrand and J. Meunier, "Ultrasonic texture motion analysis: Theory and simulation," IEEE Trans. Med. Imag., vol. 14, no. 6, pp. 293-300, Jun. 1995.

[23] J. Barron, D. Fleet, S. Beauchemin, and T. Burkitt, "Performance of optical flow techniques," Intl. J. Comput. Vis., vol. 12, pp. 43-77, 1994.

[24] S. Van Huffel and J. Vandewalle, The Total Least Squares Problem: Computational Aspects and Analysis. Philadelphia, PA: SIAM, 1991, vol. 9, Frontiers in Applied Mathematics.

[25] G. Golub and C. Van Loan, "An analysis of the total least squares problem," SIAM J. Numer. Anal., vol. 17, no. 6, pp. 883-893, Dec. 1980.

[26] M. Cerqueira, N. Weissman, V. Dilsizian, A. Jacobs, S. Kaul, W. Laskey, D. Pennell, J. Rumberger, T. Ryan, and M. Verani, "Standardized myocardial segmentation and nomenclature for tomographic imaging of the heart," Circulation, vol. 105, pp. 539-542, 2002.

[27] M. Sonka and J. Fitzpatrick, Eds., Handbook of Medical Imaging. Bellingham, WA: SPIE, 2000, vol. 2, Echocardiography, ch. 11.

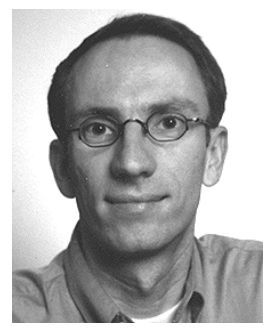

Michael Sühling (S'03) was born in Bocholt, Germany, in 1974. He received the diploma degree from the University of Kaiserslautern, Kaiserslautern, Germany, in 2000.

Currently, he is a Research Assistant with the Biomedical Imaging Group, Swiss Federal Institute of Technology Lausanne, Lausanne, Switzerland. His research interests include multiresolution image processing, wavelets, and motion analysis.

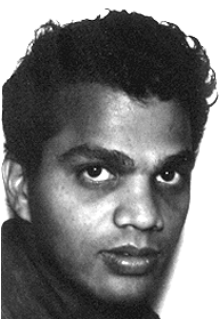

Muthuvel Arigovindan was born in Pondicherry, India, in 1974. He received the B.Tech. degree in electronics and communication engineering from Pondicherry Engineering College in 1995 and the M.Sc. degree from the Indian Institute of Science in 1999, where he worked on multirate signal processing. $\mathrm{He}$ is currently pursuing the Ph.D. degree in the Biomedical Imaging Group, Swiss Federal Institute of Technology Lausanne, Lausanne, Switzerland, where he is working on variational methods for the reconstruction of multidimensional signals from nonuniform samples.

His areas of interest include multirate signal processing, biomedical image processing, inverse problems, and numerical algorithms.

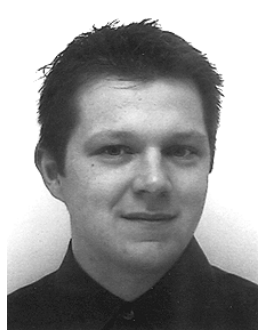

Christian Jansen was born in Orsoy, Germany, in 1970. He received the medical doctor degree from the Aachen University of Technology, Aachen, Germany, in 1999.

He currently specializes in internal medicine and cardiology. At present, he is a Research Assistant with the Physics in Medicine Group, University Hospital of Basel, Basel, Switzerland. His research interests include multimodal data processing, especially the classification of higher dimensional echocardiographic ultrasound data.

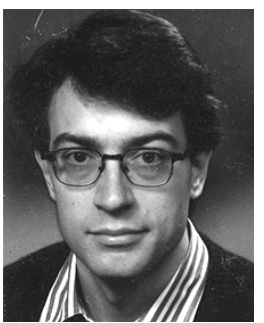

Patrick Hunziker was born in Zurich, Switzerland, on May 22, 1963. He received the M.D. degree in 1988 and subsequently specialized in internal medicine, cardiology, and intensive care medicine.

$\mathrm{He}$ is currently a Lecturer at the University of Basel, Basel, Switzerland, where he is a Clinician in the cardiac care unit and leads a research group focusing on the application of mathematics and physics in medicine. His research interests include medical data processing, especially of higher dimensional image data, as well as the application of nanotechnology in clinical medicine.

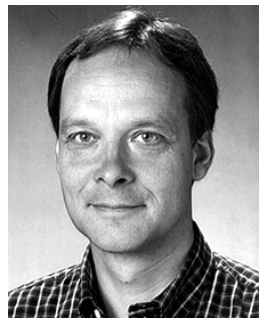

Michael Unser (M'89-SM'94-F'99) received the M.S. (summa cum laude) and Ph.D. degrees in electrical engineering from the Swiss Federal Institute of Technology Lausanne (EPFL), Lausanne, Switzerland, in 1981 and 1984, respectively.

From 1985 to 1997, he was with the Biomedical Engineering and Instrumentation Program, National Institutes of Health, Bethesda, MD, where he headed the Image Processing Group. He is now Professor and Director of the Biomedical Imaging Group, EPFL. His research area is biomedical image processing. He has a strong interest in sampling theories, multiresolution algorithms, wavelets, and the use of splines for image processing, and he is the author of over 100 published journal papers in these areas.

Dr. Unser is the Associate Editor-in-Chief of the IEEE TRANSACTIONS ON MEDICAL IMAGING. He has acted as Associate Editor or member of the editorial boards for the IEEE Signal Processing Magazine, the IEEE TRANSACTIONS ON IMAGE PROCESSING (1992 to 1995), and the IEEE SigNAL PROCESSING LETTERS (1994 to 1998). He was general Co-Chair for the first IEEE International Symposium on Biomedical Imaging (ISBI'2002), Washington, DC, July 7-10, 2002. He received the 1995 and 2003 Best Paper Awards and the 2000 Magazine Award from the IEEE Signal Processing Society. 